\title{
Serum Antioxidant Levels Associated with Subcortical Ischemic Vascular Disease
}

\author{
Xia Zhou, Long Wang, Han Liu, Chao Zhang, Minmin Zhu, Xiaoqun Zhu, \\ Jiahu Hao, Zhongwu Sun
}

\begin{abstract}
Objective: We aimed to examine changes in serum bilirubin and uric acid (UA) levels in subcortical ischemic vascular disease (SIVD). In addition, we investigated if altered serum bilirubin and UA levels correlate with the subtypes of SIVD as well as the severity of leukoaraiosis (LA). Methods: This cross-sectional study included 1098 consecutive patients with slight symptoms, such as dizziness, vertigo etc. According to magnetic resonance imaging (MRI) appearances, they were divided into either SIVD group or controls $(\mathrm{CN})$, and the SIVD group was further grouped in lacunar infarction (LI) and LA subtypes, as well as different grades. Serum bilirubin and UA levels were determined by the vanadate oxidase method and enzymatic method respectively, after at least an eight hour overnight fasting, in all subjects. Results: The bilirubin level was obviously lower while the UA level was significantly higher in the SIVD group when compared with the controls. Moreover, the LA subgroup presented more significant changes in bilirubin and UA when compared to the LI subgroup in both males and females. The correlation was positive between the UA levels and the LA severity $(r=0.134, p=0.006)$. Multivariate regression analysis revealed that the odds ratio $(95 \% \mathrm{CI})$ for SIVD in the lowest tertile of total bilirubin

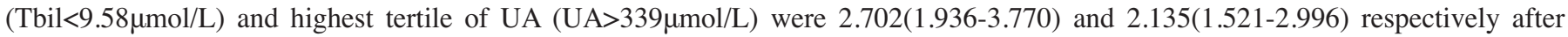
adjusting for confounding variables. Conclusion: Serum bilirubin levels were lower, whereas UA levels were higher in SIVD patients when compared with controls in both males and females, especially in LA patients. Moreover, serum UA levels positively correlated to LA severity.
\end{abstract}

RÉSUMÉ: Niveaux sériques d'antioxydants associés à la maladie vasculaire ischémique sous-corticale. Objectif : Le but de l'étude était d'examiner les changements de niveaux de la bilirubine sérique et de l'acide urique chez les patients atteints d'une maladie vasculaire ischémique souscorticale (MVIS). De plus, nous avons cherché à déterminer si une perturbation des niveaux de bilirubine sérique et d'acide urique était corrélée aux sous-types de MVIS ainsi qu'à la sévérité de la leucoaraïose. Méthode : Mille quatre-vingt-dix-huit patients consécutifs présentant des symptômes légers comme des étourdissements, du vertige et d'autres symptômes de cette nature ont été inclus dans cette étude transversale. Les patients ont été répartis en 2 groupes, soit le groupe témoin et le groupe MVIS selon les constatations faites à l'imagerie par résonance magnétique (IRM). Le groupe MVIS a été subdivisé selon que les patients présentaient un infarctus lacunaire (IL) ou de la leucoaraïose (LA) et selon le degré d'atteinte. Les niveaux de bilirubine sérique et d'acide urique (AU) ont été déterminés après un jeune de 8 heures par la méthode utilisant le vanadate comme agent oxydant et par la méthode enzymatique respectivement chez tous les sujets. Résultats : Le niveau de bilirubine était évidemment plus bas et le niveau d'acide urique était significativement plus élevé dans le groupe MVIS par rapport au groupe témoin. De plus, le sous-groupe LA avait des changements plus significatifs des niveaux de bilirubine et d'acide urique par rapport au sous-groupe atteint d'IL, tant chez les hommes que chez les femmes. La corrélation entre les niveaux d'acide urique (AU) et la sévérité de la LA était positive $(\mathrm{r}=0,134, \mathrm{p}=0,006)$. L'analyse de régression multivariée a montré que le rapport de cotes (IC à 95\%) pour la MVIS lorsque la bilirubine totale (bilT < 9,58 $\mu \mathrm{mol} / \mathrm{L}$ ) était dans le tiers inférieur de la distribution et que l'AU (AU >339 $\mu \mathrm{mol} / \mathrm{L})$ se situait dans le tiers supérieur de la distribution était de 2,702 $(1,936$ à 3,770$)$ et 2,135 $(1,521$ à 2,996$)$ respectivement, après ajustement pour les variables confondantes. Conclusion : Les niveaux de bilirubine sérique étaient plus bas alors que les niveaux d'AU étaient plus élevés chez les patients atteints de MVIS par rapport aux témoins, tant chez les hommes que chez les femmes, particulièrement chez les patients atteints de LA. De plus, les niveaux d'AU sérique étaient corrélés positivement à la sévérité de la LA.

Can J Neurol Sci. 2014; 41: 375-381

Vascular dementia (VaD) is deemed as the second most prevalent type of dementia ${ }^{1}$. Subcortical ischemic vascular disease (SIVD) comprises leukoaraiosis (LA) and lacunar infarction (LI), which is caused by chronic hypo-perfusion or small vascular occlusion. Subcortical ischemic vascular disease is the most common cause of $\mathrm{VaD}^{2}$ and is associated with the risk of stroke ${ }^{3}$. With this background, the diagnosis and interference of SIVD has received extensive attention. Increasing evidence
From the Department of Neurology, The First Affiliated Hospital of Anhui Medicial University, Anhui Province, People's Republic of China.

Received September 27, 2013. Final Revisions Submitted December 10, 2013. Correspondence to: Zhongwu Sun, Department of Neurology, The First Affiliated Hospital of Anhui Medical University, 218 Jixi Road, Anhui 230022, Anhui Province, People's Republic of China 230022. Email:sunzhwu@hotmail.com. 
suggests that oxidation plays an important role in the pathogenesis of $\mathrm{SIVD}^{4,5}$. Cerebral tissue has a low antioxidant content, contains high levels of polyunsaturated fatty acids in neuronal membranes and requires high oxygen consumption ${ }^{6}$. Because of this weakness, once the anti-oxidation capacity decreases or an excess of reactive oxygen species (ROS) is produced, cerebral tissue can easily be damaged.

Bilirubin is an end product of heme metabolism ${ }^{7}$. Some investigations indicate that elevated serum bilirubin levels are associated with increased stroke severity and may reflect the intensity of oxidative stress ${ }^{8}$. However, a growing body of evidence supports the theory that bilirubin harbors powerful antioxidant and anti-inflammatory properties ${ }^{9}$. Some clinical studies indicated that it plays a beneficial role in oxidative stressmediated disease, such as coronary heart disease and stroke ${ }^{10}$. Interestingly, these potentially contradictory properties have also been observed with another end product, uric acid (UA), which is an inert byproduct of purine metabolism. Uric acid may confer a protective role in acute ischemic stroke and degeneration disorder, such as Alzheimer disease (AD) ${ }^{11}$ and Parkinson disease $^{12}$, as a consequence of its antioxidant properties. On the other hand, several lines of evidence suggest that elevated serum UA predicts the occurrence of myocardial infarction ${ }^{13}$ and metabolic syndrome ${ }^{14}$. One source even indicated no association between UA and acute ischemic stroke outcomes, regardless of short or long term injury ${ }^{15}$. In this case, we should consider that bilirubin and UA, with contradictory properties, contribute to the pathophysiology of brain injury and/or protect the brain against injury.

Because of their biological effect, a greater number of investigations are focusing on the change of serum bilirubin and UA in oxidative stress-mediated disease, especially in cardiocerebrovascular disease. However, there is little evidence regarding the distribution of serum bilirubin and UA in patients with SIVD, and no studies have investigated the different kinds of bilirubin in the two subtypes of SIVD or focused on the different severities of LA.

In this study, we aimed to examine changes in serum bilirubin and UA levels in SIVD. In addition, we investigated if altered serum bilirubin and UA levels correlate with the subtypes of SIVD as well as the severity of LA.

\section{METHODS}

\section{Subjects and diagnosis criteria}

In this retrospective study, we analyzed 1098 consecutive patients with slight symptoms, such as dizziness, vertigo, diplopia, lumbar disk disease and insomnia in the First Affiliated Hospital of Anhui Medical University from January 2010 to December 2012. According to magnetic resonance imaging (MRI) appearances, they were divided into either SIVD group or controls $(\mathrm{CN})$. This study was approved by the ethics committee of Anhui Medical University and informed consent was obtained from all participants.

Study entry criteria were: (1) SIVD was defined as subcortical white matter hyperintensity or lacunar infarct on T2weighted images, and/or fluid attenuated inversion recovery (FLAIR) images and without any acute stroke appearances in diffusion weighted imaging (DWI). (2) Controls were subjects without both the LA and LI in MRI and without any cognition or emotional complaints such as memory, processing, visual-spatial dysfunction, depression etc.

Subjects meeting any of the following criteria were excluded: hepatitis B surface antigen evidence or hepatitis $\mathrm{C}$ virus antibody, a history of chronic liver disease or abnormal liver function [aspartate aminotransferase (AST) or alanine aminotransferase $($ ALT) $>40 \mathrm{U} / \mathrm{L}]$, renal failure, having signs of secondary inflammatory conditions, previous corticosteroid treatment, having a high-fat diet or seafood three days before enrolment.

\section{Clinic basis and laboratory measurements}

All subjects were questioned for a history of hypertension (known hypertension treated with antihypertensive drugs, two or more blood pressure recordings higher than 140/90 $\mathrm{mmHg}$ on different days taken after a 15 minute ( $\min )$ resting period), diabetes mellitus (known diabetes treated with diet or drugs or a fasting plasma glucose level $\geq 7.0 \mathrm{mmol} / \mathrm{L}$ ), ischemic heart disease (known ischemic heart disease treated with improving myocardial ischemic drugs or having a history of coronary heart disease), smoking and alcohol consumption history. The body mass index (BMI) was calculated by dividing the weight of the patient in kilograms by the square of the height in meters. After at least an eight hour overnight fasting, blood samples were obtained between 6:30 and 8:30 am from all subjects. The UA concentration was measured using the direct enzymatic method, where UA was oxidized by uricase coupled with peroxidase. Serum bilirubin concentrations, including directed bilirubin (Dbil), indirected bilirubin (Ibil), and total bilirubin (Tbil) were measured using the vanadate oxidase method. Both tests were measured using a Modular DPP AYL-5-001 autoanalyzer (Roche, Switzerland). Simultaneously, AST, ALT, blood urea nitrogen (BUN), creatinine $(\mathrm{Cr})$, fasting blood glucose (FBG), levels of total cholesterol (TC), low-density lipoprotein cholesterol (LDL-C), high-density lipoprotein cholesterol (HDL-C), and triglyceride (TG) were measured by the enzymatic method on the same analyzer.

Brain MRI was performed using a 3.0-Tesla Signa Horizon (GE Medical Systems, Harvey, IL, USA) to obtain T1-, T2weighted images, FLAIR images and DWI in all subjects. Magnetic resonance imaging features were evaluated by an experienced radiologist who was unaware of the aims of the study and blinded to laboratory findings.

\section{SIVD subtypes}

To explore possible pathogenic differences, SIVD patients were further subtyped into two groups: LA (lacunar infarction with confluent leukoaraiosis) and LI (lacunar infarction with absent or mild leukoaraiosis) according to their MRI scan appearances using a semiquantitative rating scale. Leukoaraiosis were rated as: grade 0 , none (absent); grade 1 , mild white matter hyperintensities (mild); grade 2, moderate confluent (moderate); and grade 3, severe confluent (severe), according to the Fazekas scale $^{16}$.

\section{Statistical analysis}

Continuous variables in this study were presented as the means \pm standard deviation (SD). Statistical significance was set 
at $p \geq 0.05$. Two-sample two-tailed t-tests were used for continuous variables and chi-square tests for categorical variables between the SIVD group and controls. Because serum bilirubin and UA levels have been shown to be dependent on age and gender, the analysis of covariance (ANCOVA) was used to compare serum bilirubin and UA levels in patients among LA, LI and the controls with age as a covariant stratified by gender. The ANCOVA analysis was used for age, gender and vascular risk factor controls in their associations with the serum bilirubin, UA and LA severity. The Pearson correlation coefficient (r) was calculated for the correlation between bilirubin and UA levels and LA severities. To assess the relationship between Tbil, UA levels and the risk of SIVD, Tbil and UA levels were divided into tertiles and 95\% confidence intervals (95\% CI) for SIVD were calculated using multiple stepwise logistic regression analysis after adjusting for confounding variables (age, gender, BMI, hypertension, diabetes, ischemic heart disease, smoking and alcohol consumption, HDL, LDL, TG, TC, FBG, BUN, Cr) across the tertiles. All statistical analyses were performed using the Statistical Program for Social Sciences statistical software (version 16.0 SPSS).

\section{Results \\ Subject characteristics}

A total of 733 patients with SIVD [males: 343, females: 390] and 365 healthy controls [males: 159, females: 206] were evaluated. The demographic properties and laboratory parameters are presented in Table 1. Subcortical ischemic vascular disease was frequently associated with hypertension and old age. The HDL-C levels were significantly lower in SIVD compared with the controls. The bilirubin level, including Tbil, Dbil and Ibil, was obviously lower while the UA level was significantly higher in the SIVD group when compared with the controls $(p<0.05$; Table 1).

\section{Bilirubin and UA in SIVD subtype}

The differences between SIVD and controls in bilirubin and UA levels were also investigated when the SIVD group was separated into LA and LI in both males and females. The serum bilirubin level was lowest and the UA level was highest in LA, while LI moderately decreased or increased in comparison with the controls. The difference in bilirubin and UA levels between the LA and LI groups reached statistical significance $(p<0.05$; Table 2, Figure 1).

Table 1: Baseline clinical characteristics of the subjects (Mean \pm SD or $\mathbf{n}(\%)$ )

\begin{tabular}{|c|c|c|c|}
\hline Variables & $\operatorname{SIVD}(\mathrm{n}=733)$ & $\mathrm{CN}(\mathrm{n}=365)$ & $\mathrm{p}$ values \\
\hline Age & $68.54 \pm 8.60$ & $65.75 \pm 8.00$ & $<0.001$ \\
\hline Male, no.(\%) & $343(46.8)$ & $159(43.6)$ & 0.171 \\
\hline Height (m) & $1.64 \pm 0.68$ & $1.64 \pm 0.67$ & 0.779 \\
\hline Weight (kg) & $62.92 \pm 8.32$ & $62.13 \pm 8.33$ & 0.767 \\
\hline Body mass index $\left(\mathrm{kg} / \mathrm{m}^{2}\right)$ & $23.07 \pm 2.51$ & $22.97 \pm 2.46$ & 0.539 \\
\hline Hypertension $^{\mathrm{a}}$, no.(\%) & $454(61.9)$ & $158(43.3)$ & $<0.001$ \\
\hline Diabetes Mellitus ${ }^{\mathrm{b}}$, no.(\%) & $137(18.7)$ & $64(17.5)$ & 0.679 \\
\hline Coronary artery disease, no.(\%) & $50(6.8)$ & $33(9.0)$ & 0.225 \\
\hline Current smoker, no.(\%) & $113(15.4)$ & $70(19.2)$ & 0.069 \\
\hline Alcohol consumptiom ${ }^{\mathrm{c}}$, no.( $\left.\%\right)$ & $78(10.6)$ & $49(13.4)$ & 0.105 \\
\hline Direct bilirubin (Dbil, $\mu \mathrm{mol} / \mathrm{L})$ & $3.37 \pm 1.38$ & $3.82 \pm 1.67$ & $<0.001$ \\
\hline Indirect bilirubin (Ibil, $\mu \mathrm{mol} / \mathrm{L}$ ) & $7.96 \pm 3.55$ & $9.47 \pm 4.00$ & $<0.001$ \\
\hline Total bilirubin (Tbil, $\mu \mathrm{mol} / \mathrm{L}$ ) & $11.27 \pm 4.50$ & $13.35 \pm 5.21$ & $<0.001$ \\
\hline Uric acid $(\mathrm{UA}, \mu \mathrm{mol} / \mathrm{L})$ & $316.12 \pm 83.31$ & $283.36 \pm 75.39$ & $<0.001$ \\
\hline High-density lipoprotein cholesterol (HDL-C, mmol/L) & $1.25 \pm 0.34$ & $1.33 \pm 0.38$ & $<0.001$ \\
\hline Low-density lipoprotein cholesterol (LDL-C, mmol/L) & $2.84 \pm 0.86$ & $2.81 \pm 0.87$ & 0.651 \\
\hline Total cholesterol (TC, mmol/L) & $4.74 \pm 1.03$ & $4.74 \pm 1.02$ & 0.991 \\
\hline Triglycerides (TG, mmol/L) & $1.43 \pm 0.64$ & $1.36 \pm 0.52$ & 0.122 \\
\hline Fasting plasma glucose $(\mathrm{FBG}, \mathrm{mmol} / \mathrm{L})$ & $6.20 \pm 1.52$ & $5.88 \pm 1.28$ & 0.171 \\
\hline Blood urea nitrogen (BUN, mmol/L) & $5.64 \pm 1.75$ & $5.32 \pm 1.47$ & 0.003 \\
\hline Creatinine $(\mathrm{Cr}, \mu \mathrm{mol} / \mathrm{L})$ & $67.80 \pm 19.85$ & $66.63 \pm 21.4$ & 0.367 \\
\hline
\end{tabular}

${ }^{\mathrm{a}}$ Hypertension was defined as at least twice systolic blood pressure (SBP) $\geq 140 \mathrm{mmHg}$, diastolic blood pressure $(\mathrm{DBP}) \geq 90 \mathrm{mmHg}$ in different days taken after 15 -min resting periods, or a history of the disorder; biabetes was defined as fasting plasma glucose level $\geq 7.0 \mathrm{mmol} / 1$ or a history of the disorder; ${ }^{\mathrm{c}}$ Alcohol consumption $\geq 140 \mathrm{~g} /$ week; SIVD: subcortical ischemic vascular disease; $\mathrm{CN}$ :controls 


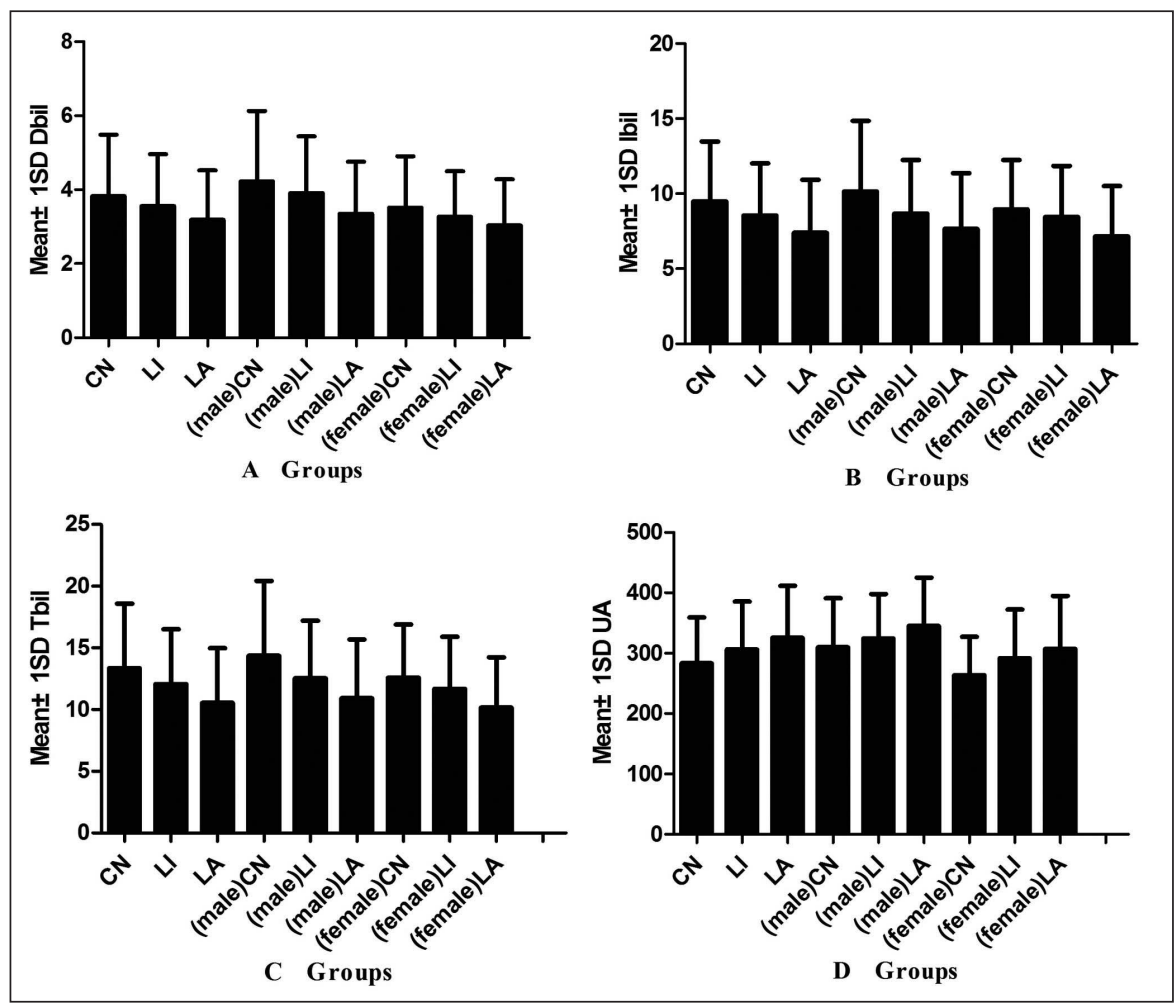

Figure 1: The comparison of direct bilirubin (A), indirect bilirubin (B), total bilirubin $(C)$ and uric acid $(D)$ between healthy controls and patients with lacunar infarction and leukoaraiosis. LA:leukoaraiosis; LI:lacunar infarction; CN:controls

Table 2: Serum bilirubin and UA in controls and patients with LI and LA

\begin{tabular}{clll}
\hline Variables & Total & Male & Female \\
\hline Dbil $(\mu \mathrm{mol} / \mathrm{L})$ & & & \\
LA & $3.19 \pm 1.34^{\mathrm{ab}}$ & $3.35 \pm 1.41^{\mathrm{ab}}$ & $3.03 \pm 1.25^{\mathrm{a}}$ \\
LI & $3.56 \pm 1.40^{\mathrm{a}}$ & $3.91 \pm 1.53$ & $3.29 \pm 1.24$ \\
CN & $3.82 \pm 1.67$ & $4.22 \pm 1.90$ & $3.51 \pm 1.41$ \\
Ibil $(\mu \mathrm{mol} / \mathrm{L})$ & & & \\
LA & $7.40 \pm 3.54^{\mathrm{ab}}$ & $7.64 \pm 3.72^{\mathrm{ab}}$ & $7.16 \pm 3.35^{\mathrm{ab}}$ \\
LI & $8.55 \pm 3.47^{\mathrm{a}}$ & $8.67 \pm 3.56^{\mathrm{a}}$ & $8.46 \pm 3.39$ \\
CN & $9.74 \pm 4.00$ & $10.14 \pm 4.71$ & $8.96 \pm 3.30$ \\
Tbil $(\mu \mathrm{mol} / \mathrm{L})$ & & & $10.16 \pm 4.07^{\mathrm{ab}}$ \\
LA & $10.53 \pm 4.43^{\mathrm{ab}}$ & $10.92 \pm 4.76^{\mathrm{ab}}$ & $11.67 \pm 4.25^{\mathrm{a}}$ \\
LI & $12.06 \pm 4.40^{\mathrm{a}}$ & $12.53 \pm 4.65^{\mathrm{a}}$ & $12.57 \pm 4.31$ \\
CN & $13.35 \pm 5.22$ & $14.37 \pm 6.06$ & \\
UA $(\mu \mathrm{mol} / \mathrm{L})$ & & & $306.92 \pm 87.74^{\mathrm{a}}$ \\
LA & $325.51 \pm 86.12^{\mathrm{ab}}$ & $345.02 \pm 80.08^{\mathrm{ab}}$ & $291.55 \pm 80.81^{\mathrm{a}}$ \\
LI & $306.28 \pm 79.20^{\mathrm{a}}$ & $324.53 \pm 73.39$ & $263.44 \pm 63.94$ \\
CN & $305.32 \pm 82.17$ & $309.81 \pm 81.05$ & \\
\hline
\end{tabular}

a: LA or LI VS. CN, p<0.05; b: LA VS. LI, p<0.05; Dbil: direct bilirubin; Ibil: indirect bilirubin; Tbil: total bilirubin; UA: uric acid; LA:leukoaraiosis; LI:lacunar infarction; CN:controls 


\section{Association between bilirubin, UA and LA severity in SIVD}

We demonstrated a positive relationship between elevated UA levels and LA severity ( $r=0.134, p=0.006)$. After adjusting for age, gender and vascular risk factors, there were significant differences between moderate and severe LA compared with absent LA patients in Dbil and Tbil $(p<0.05)$ as well as in UA levels $(p<0.001$; Figure 2$)$.

\section{Multivariate logistic stepwise regression}

In comparison to the subjects in the reference tertile (Tbil: $>12.99 \mu \mathrm{mol} / \mathrm{L})$, the odds ratio $(95 \% \mathrm{CI})$ for LA in the 2 nd tertile (Tbil: $9.58-12.99 \mu \mathrm{mol} / \mathrm{L})$ was $1.729(1.261-2.371)$ and in the 3rd tertile (Tbil:<9.58 $\mu \mathrm{mol} / \mathrm{L})$ was $2.702(1.936-3.770)$ after adjusting for confounding variables. With respect to UA, the odds ratio $(95 \% \mathrm{CI})$ for LA in the 2nd tertile (UA: 267-339 $\mu \mathrm{mol} / \mathrm{L}$ ) was $1.548(1.127-2.127)$ and in the 3 rd tertile (UA>339 $\mu \mathrm{mol} / \mathrm{L})$ was 2.135 (1.521-2.996) when compared with the reference tertile (UA: $<267 \mu \mathrm{mol} / \mathrm{L})$ (Table 3).

\section{Discussion}

In this study, we observed a significantly lower serum bilirubin level in SIVD compared with controls, while UA levels were notably higher in the SIVD group. When comparing the two subgroups of SIVD and controls, we found serum bilirubin levels to be lower whereas UA levels were higher in LA patients, and a moderate decrease or increase was seen in the isolated lacunar infarct group. Furthermore, there was a strong correlation between decreased bilirubin as well as elevated UA levels and LA severity, which suggests that either bilirubin or UA levels are a very strong risk factor for the development of LA. To our knowledge, this is the first report to identify an association between different subgroups of SIVD and both bilirubin and UA levels.

Apart from the traditional vascular pathologies, including hypertension, diabetes mellitus and hyperlipidemia, increasing evidence $^{4-5}$ indicates the novel vascular risk factors of inflammation, platelet activation and especially oxidative stress, to play a critical role in the pathologies of SIVD. Also, impaired vascular tone and endothelial dysfunction, to some degree, may contribute to the development of ischemic changes ${ }^{17}$, eventually permitting cerebrospinal fluid to cross the blood-brain barrier and allow interstitial water to accumulate, resulting in edema areas identified as white matter hyperintensity or lacunar infarction on brain MRI ${ }^{16}$.

Bilirubin was believed to be a waste product, and a potentially toxic compound. However, recent data have convincingly demonstrated its beneficial roles in cerebrovascular disease ${ }^{10}$. To the best of our knowledge, only one study has compared total bilirubin levels in SIVD patients ${ }^{18}$. Similarly, serum bilirubin levels in patients with SIVD were lower when compared with controls in the present study, irrespective of the kind of bilirubin. Moreover, we further compared three kinds of bilirubin levels in different subtypes of SIVD. The decrease in bilirubin levels remained significant in both LA and LI subtypes. Moreover, bilirubin levels were slightly lower in LA compared with LI in both males and females. Accumulating evidence suggests that bilirubin plays an important role in inhibiting the

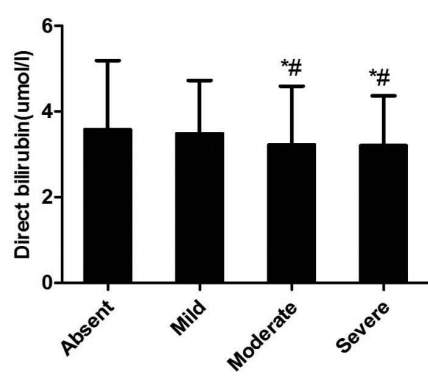

A Leukoraiosis grade

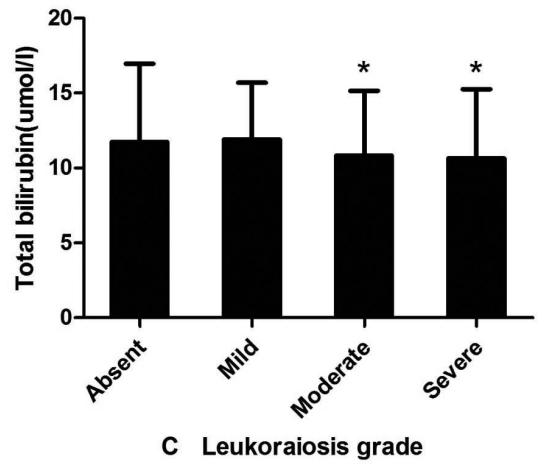

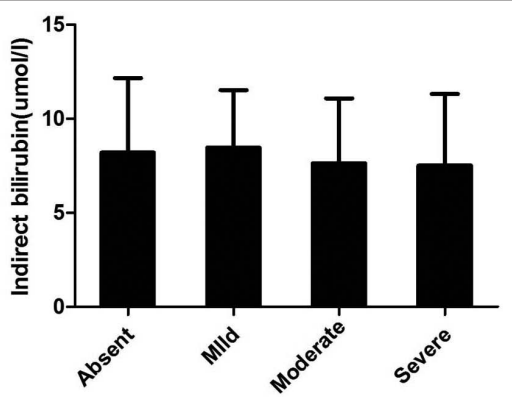

B Leukoraiosis grade

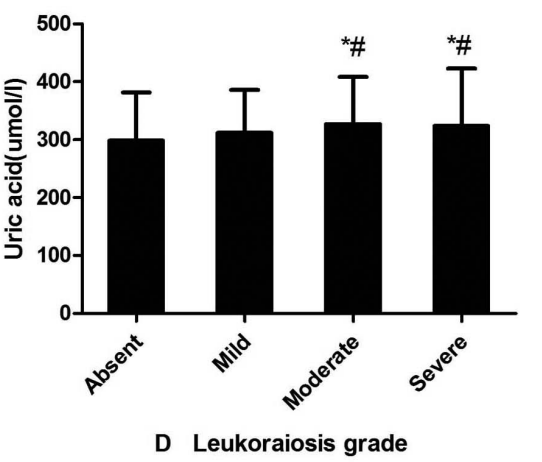

Figure 2: Mean direct bilirubin (A), indirect bilirubin $(B)$, total bilirubin $(C)$ and uric acid $(D)$ levels in patients with different grade leukoaraiosis. $p$ value were given for significant difference compared to the absent grade. ${ }^{*} p<0.05, \# p<0.01$. 
Table 3: Multiple logistic stepwise regression analysis results of the possible correlation for SIVD

\begin{tabular}{lllll}
\hline Variables & Beta estimate & odds ratio & $95 \%$ CI & p value \\
\hline Hypertension & 0.666 & 1.946 & $1.490-2.541$ & $<0.001$ \\
Age & 0.035 & 1.036 & $1.019-1.053$ & $<0.001$ \\
HDL-C & -0.398 & 0.672 & $0.460-0.981$ & 0.039 \\
Tbil $(<9.58 \mu \mathrm{mol} / \mathrm{L})$ & 0.994 & 2.702 & $1.936-3.770$ & $<0.001$ \\
$\operatorname{Tbil}(9.58-12.99 \mu \mathrm{mol} / \mathrm{L})$ & 0.548 & 1.729 & $1.261-2.371$ & $<0.001$ \\
$\operatorname{Tbil}(>12.99 \mu \mathrm{mol} / \mathrm{L})^{*}$ & - & - & - & - \\
$\mathrm{UA}(<267 \mu \mathrm{mol} / \mathrm{L})^{*}$ & - & - & - & - \\
$\mathrm{UA}(267-339 \mu \mathrm{mol} / \mathrm{L})$ & 0.473 & 1.548 & $1.127-2.127$ & 0.007 \\
$\mathrm{UA}(>339 \mu \mathrm{mol} / \mathrm{L})$ & 0.758 & 2.135 & $1.521-2.996$ & $<0.001$ \\
\hline
\end{tabular}

*Reference group. HDL-C: High-density lipoprotein cholesterol; Tbil: total bilirubin; UA: uric acid; SIVD: subcortical ischemic vascular disease

oxidation of LDL cholesterol and monocyte migration ${ }^{19}$ as well as vascular smooth muscle cell proliferation ${ }^{20}$, thus having a protective effect on SIVD. Moreover, Erdogan et $a l^{21}$ indicated that even in healthy people, lower serum bilirubin levels were independently related to endothelial dysfunction and increased carotid intima-media thickness, suggesting a protective effect of serum bilirubin on endothelium and atherosclerosis. Additionally, some production molecules related to bilirubin metabolism have been recently proposed to contribute to the protective effect against oxidative stress, such as heme oxygenize (HO) and biliverdin ${ }^{22}$. It is mandatory to mention another important mechanism of bilirubin, which is to contribute to the reduced risk of SIVD. This role involves the significant inhibition of collagen induced platelet activation ${ }^{23}$, thus helping to reduce the risk associated with intravascular thrombosis.

It is commonly believed that UA is the most abundant aqueous antioxidant in humans, while oxidative stress may contribute to the pathology of SIVD. It may therefore be expected that the UA level of SIVD would be lower than those of normal controls. However, a rather unexpected result was that UA levels were significantly elevated in SIVD when compared with the controls. Likewise, no definite explanation could be provided for the increase in UA levels in SIVD patients. Nevertheless, several possible explanations may be proposed to explain the apparent paradox in SIVD. Elevated UA levels may accelerate platelet adhesiveness ${ }^{24}$, vascular smooth muscle cell proliferation and oxidation of $\mathrm{LDL}^{25}$ and are inversely proportional to nitric oxide levels, a potent vasodilator, which subsequently results in impaired vascular tone and cerebral ischemia. Uric acid can become a pro-oxidant under certain circumstances, particularly if other antioxidant substances such as ascorbate are low $^{26}$. Moreover, a direct neurotoxic effect of UA has been reported in vitro ${ }^{27}$, while elevated UA levels in vivo have been deemed to be closely related to vascular risk factors, such as hypertension ${ }^{28}$ and diabetes mellitus ${ }^{29}$. The interaction between UA and vascular risk factors may also play a critical role in the development of SIVD. On the other hand, given that
UA plays beneficial roles in $\mathrm{AD}$ and stroke via its anti-oxidation capability, it also appears plausible that UA may be an evolutionary antioxidant substitute for the loss of ability to synthesize other antioxidants against free radical damage. However, this point is supported by few studies, suggesting that hyperuricemia may be a compensatory measure to counteract the oxidative stress damage related to atherosclerosis ${ }^{30}$.

Consistently, in 2007, Schretlen et $a l^{31}$ cross-sectionally examined 177 adults, and demonstrated that elevated serum UA was significantly associated with subcortical white matter hyperintensities after controlling for confounds. Furthermore, they also showed that even mild elevation in serum UA is associated with cerebral ischemia ${ }^{32}$, which finally causes white matter impairment. In addition, serum UA levels have been shown to be associated with several other ischemic diseases ${ }^{33,34}$. Previous meta-analyses ${ }^{33}$ revealed that hyper-uricemia may marginally increase the risk of coronary heart disease events, independent of traditional coronary heart disease risk factors, and elevated serum UA levels were suggested to predict the occurrence of stroke, subsequent vascular events following an initial stroke and fatal cardiovascular events ${ }^{34}$. Moreover, similar to bilirubin, the change in UA levels was more dramatic in LA patients than those of LI. Both clinical and pathological studies support the fact that lacunar infarction and leukoaraiosis represent different forms of small vessel disease. It has been demonstrated that localized microatheroma at the origin of perforating arterioles may partly be responsible for some cases of isolated lacunar infarction, while diffuse arteriosclerosis is the predominant pathology in the LA group, which is deemed similar to that observed in the penumbra of large infarcts, producing a large amount of $\operatorname{ROS}^{35}$. Consistent with this heterogeneity, we found differing associations between LI and LA in both bilirubin and UA. Our findings also support and expand on a previous study ${ }^{31}$ examining the relationship between serum UA, bilirubin and the severity of white matter lesions. The highest UA levels and lowest bilirubin levels were found in SIVD patients with severe LA, which implicates the 
underlying pathophysiology of UA and bilirubin in SIVD development. This obligates us to pay special attention to people with abnormal UA and bilirubin levels.

We acknowledge the following limitations in our study. First, our study design is a cross-sectional study, which is not the best way to investigate any causal relationships, and additional studies are needed to establish cause and effect between serum bilirubin, UA and SIVD. Second, both SIVD patients and the controls were confined to the hospital, which made it impossible to correlate our data to the general population of SIVD and normal personnel. Third, we did not analyze the number of LI and genetic susceptibility factors in subjects, which may be considered as confounding factors, influencing the result to some extent.

Although the background bilirubin level decreases and UA level increases have yet to be investigated, a therapy to increase bilirubin and/or to reduce UA levels is a possible option for the treatment of SIVD as a consequence of bilirubin anti-oxidation behavior and UA pro-oxidation risk. In the future, serum bilirubin and UA levels may serve as effective tools to identify elders at risk of developing SIVD, which may facilitate proactive and appropriate interventions to prevent functional decline.

\section{CONCLUSION}

In summary, serum bilirubin levels were lower, whereas UA levels were higher in SIVD patients when compared with controls in both males and females, especially in LA patients. Moreover, serum UA levels positively correlated to LA severity.

\section{ACKNOWLEDGEMENTS}

This study was supported by grants from Natural Science Foundation of China in Anhui Province Department of Education (KJ2011A170).

\section{REFERENCES}

1. Staekenborg SS, Su T, van Straaten EC, et al. Behavioural and psychological symptoms in vascular dementia; differences between small- and large-vessel disease. J Neurol Neurosurg Psychiatry. 2010;81(5):547-51.

2. Erkinjuntti T. Subcortical vascular dementia. Cerebrovasc Dis. 2002;13 Suppl 2:58-60.

3. Jeerakathil T, Wolf PA, Beiser A, et al. Stroke risk profile predicts white matter hyperintensity volume: the Framingham Study. Stroke. 2004;35(8): 1857-61.

4. Al Ahmad A, Gassmann M, Ogunshola OO. Involvement of oxidative stress in hypoxia-induced blood-brain barrier breakdown. Microvasc Res. 2012;84(2):222-5.

5. Wengreen HJ, Munger RG, Corcoran CD, et al. Antioxidant intake and cognitive function of elderly men and women: the Cache County Study. J Nutr Health Aging. 2007;11(3):230-7.

6. Christen Y. Oxidative stress and Alzheimer disease. Am J Clin Nutr. 2000; 71(2): 621S-9S.

7. Stocker R, Yamamoto Y, McDonagh AF, et al. Bilirubin is an antioxidant of possible physiological importance. Science. 1987; 235(4792):1043-6.

8. Xu T, Zhang J, Liu W, et al. Association of serum bilirubin with stroke severity and clinical outcomes. Can J Neurol Sci. 2013; 40(1):80-4.

9. Sedlak TW, Saleh M, Higginson DS, et al. Bilirubin and glutathione have complementary antioxidant and cytoprotective roles. Proc Natl Acad Sci USA. 2009;106(13):5171-6.

10. Oda E, Kawai R. A possible cross-sectional association of serum total bilirubin with coronary heart disease and stroke in a
Japanese health screening population. Heart Vessels. 2012;27 (1):29-36.

11. Spitsin S, Koprowski H. Role of uric acid in Alzheimer's disease. J Alzheimers Dis. 2010;19(4):1337-8.

12. Shen C, Guo Y, Luo W, et al. Serum urate and the risk of Parkinson's disease: results from a meta-analysis. Can J Neurol Sci. 2013;40 (1):73-9.

13. Larina VN, Bart BIa, Larin VG, et al. [Hyperuricemia and cardiovascular continuum]. Klin Med (Mosk). 2013;91(1):11-5.

14. Tutal E, Sayin B, Ertugrul DT, et al. Is there a link between hyperuricemia, morning blood pressure surge, and non-dipping blood pressure pattern in metabolic syndrome patients? Int $\mathbf{J}$ Nephrol Renovasc Dis. 2013;6:71-7.

15. Miedema I, Uyttenboogaart M, Koch M, et al. Lack of association between serum uric acid levels and outcome in acute ischemic stroke. J Neurol Sci. 2012;319(1-2):51-5.

16. Fazekas F, Schmidt R, Scheltens P. Pathophysiologic mechanisms in the development of age-related white matter changes of the brain. Dement Geriatr Cogn Disord. 1998;9 Suppl 1:2-5.

17. Hoth KF, Tate DF, Poppas A, et al. Endothelial function and white matter hyperintensities in older adults with cardiovascular disease. Stroke. 2007;38(2):308-12.

18. Park BJ, Shim JY, Lee HR, et al. Association between serum total bilirubin level and leukoaraiosis in Korean adults. Clin Biochem. 2012;45(4-5):289-92.

19. Morita T. Heme oxygenase and atherosclerosis. Arterioscler Thromb Vasc Biol. 2005;25(9):1786-95.

20. Ollinger R, Bilban M, Erat A, et al. Bilirubin: a natural inhibitor of vascular smooth muscle cell proliferation. Circulation. 2005;112 (7): 1030-9.

21. Erdogan D, Gullu H, Yildirim E, et al. Low serum bilirubin levels are independently and inversely related to impaired flowmediated vasodilation and increased carotid intima-media thickness in both men and women. Atherosclerosis. 2006;184 (2):431-7.

22. Aztatzi-Santillan E, Nares-Lopez FE, Marquez-Valadez B, et al. The protective role of heme oxygenase-1 in cerebral ischemia. Cent Nerv Syst Agents Med Chem. 2010;10(4):310-6.

23. Kundur AR, Bulmer AC, Singh I. Unconjugated bilirubin inhibits collagen induced platelet activation. Platelets. 2014;25(1):45-50.

24. Mazzali M, Kanellis J, Han L, et al. Hyperuricemia induces a primary renal arteriolopathy in rats by a blood pressureindependent mechanism. Am J Physiol Renal Physiol. 2002;282 (6):F991-7.

25. Patterson RA, Horsley ET, Leake DS. Prooxidant and antioxidant properties of human serum ultrafiltrates toward LDL: important role of uric acid. J Lipid Res. 2003;44(3):512-21.

26. Abuja PM. Ascorbate prevents prooxidant effects of urate in oxidation of human low density lipoprotein. FEBS Lett. 1999; 446(2-3):305-8.

27. Bavaresco CS, Zugno AI, Tagliari B, et al. Inhibition of $\mathrm{Na}+\mathrm{K}+-$ ATPase activity in rat striatum by the metabolites accumulated in Lesch-Nyhan disease. Int J Dev Neurosc. 2004;22(1):11-7.

28. Feig DI. Hyperuricemia and hypertension. Adv Chronic Kidney Dis. 2012;19(6):377-85.

29. Richette P, Perez-Ruiz F. Serum uric acid and metabolic risk. Curr Med Res Opin. 2013;29 Suppl 3:9-15.

30. Nieto FJ, Iribarren C, Gross MD, et al. Uric acid and serum antioxidant capacity: a reaction to atherosclerosis? Atherosclerosis. 2000;148(1):131-9.

31. Schretlen DJ, Inscore AB, Jinnah HA, et al. Serum uric acid and cognitive function in community-dwelling older adults. Neuropsychology. 2007;21(1):136-40.

32. Schretlen DJ, Inscore AB, Vannorsdall TD, et al. Serum uric acid and brain ischemia in normal elderly adults. Neurology. 2007;69 (14):1418-23.

33. Kim SY, Guevara JP, Kim KM, et al. Hyperuricemia and coronary heart disease: a systematic review and meta-analysis. Arthritis Care Res (Hoboken). 2010; 62(2): 170-80.

34. Kim SY, Guevara JP, Kim KM, et al. Hyperuricemia and risk of stroke: a systematic review and meta-analysis. Arthritis Rheum. 2009;61(7):885-92.

35. Fisher CM. The arterial lesions underlying lacunes. Acta Neuropathol. 1968;12(1):1-15. 